\title{
AML presenting as a mediastinal mass and right ventricular failure
}

\author{
Jeremy B. Moad", Kellie R. Jones
}

Pulmonary and Critical Care Medicine, Department of Medicine, University of Oklahoma Health Sciences Center, Oklahoma City, USA; "Corresponding Author: jeremy-moad@ouhsc.edu

Received 20 January 2013; revised 27 February 2013; accepted 6 March 2013

\begin{abstract}
Extrinsic compression of the pulmonary arteries by mediastinal masses, while rare, is the most common cause of non-congenital peripheral pulmonary artery stenosis. Granulocytic myeloid sarcomas are an extramedullary manifestation of acute myeloid leukemia, with a wide variability in their presentation. They can present either de novo as soft tissue masses or in conjunction with acute myeloid leukemia. Myeloid sarcomas are uncommon and their prognosis is generally considered poor when present in patients with acute myeloid leukemia (AML). We present a case of a granulocytic myeloid sarcoma causing pulmonary artery stenosis and right ventricular failure. This case represents a unique cause of pulmonary arterial stenosis.
\end{abstract}

Keywords: Granulocytic Myeloid Sarcoma; Myeloid Sarcoma; Right Heart Failure; Pulmonary Artery Stenosis

\section{CASE PRESENTATION}

A 26-year-old previously healthy male presented to the emergency department with a three week history of progressively worsening shortness of breath. One week prior to presentation, he was experiencing orthopnea and chest pain. The chest pain was described retrosternal, stabbing, and without radiation. He reported a $10-15 \mathrm{lb}$ weight loss over 3 weeks, occasional night sweats, but no fevers or chills. His vital signs were stable, but he had a positive pulsus pardoxus of $18 \mathrm{mmHg}$. Chest xray revealed a widened mediastinum which was an acute change from a recent chest xray (see Figure 1). Labs were completely unremarkable.

Transthoracic echocardiogram revealed a large pericardial effusion along with an elevated pulmonary artery systolic pressure (PA) of $96 \mathrm{mmHg}$ and a severely reduced right ventricular systolic function. The patient

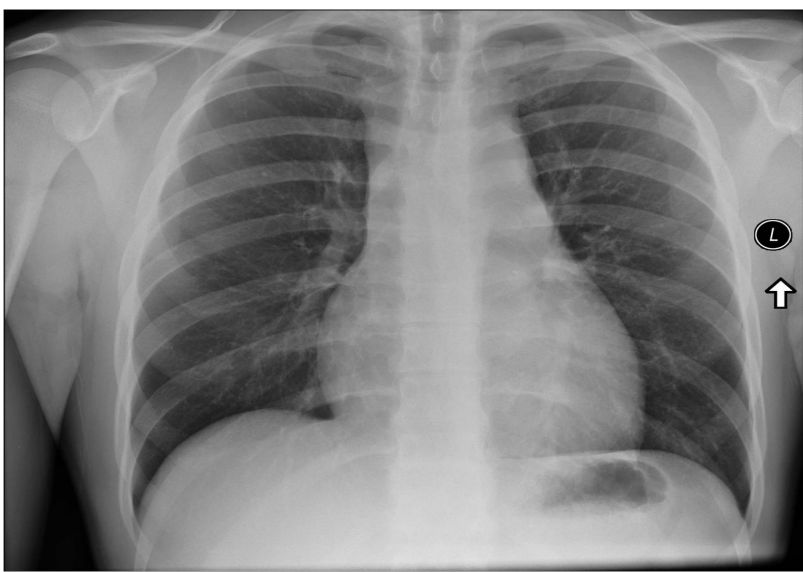

Figure 1. Chest X-ray with a widened mediastinum.

underwent a pericardiocentesis and a concurrent right heart catheterization. Approximately $600 \mathrm{cc}$ of bloody fluid was removed, and sent for cytology. Visualization during contrast infusion at the right heart catheterization suggested extrinsic compression of the main pulmonary artery.

A CT of the chest showed a heterogeneous mass present within the mediastinum with complete infiltration of the prevascular space and encasement of the ascending aorta, aortic arch, and origins of the brachiocephalic trunk, left common carotid artery, and left subclavian artery. The mass also extended posteriorly and encased and narrowed the bilateral main pulmonary arteries (see Figure 2 and arrow).

The pathology results from the pericardial fluid revealed a distinct population of blasts with myeloid features consistent with acute myeloid leukemia (see Figure 3 with Auer Rod). Flow cytometry of the bone marrow verified the findings with an 8:21 translocation and loss of the Y chromosome, indicative of the AML, despite the absence of blasts in the peripheral blood. The patient's presentation was consistent with granulocytic myeloid sarcoma involving the pericardium and causing pulmonary artery stenosis and consequently right heart failure by extrinsic compression. 


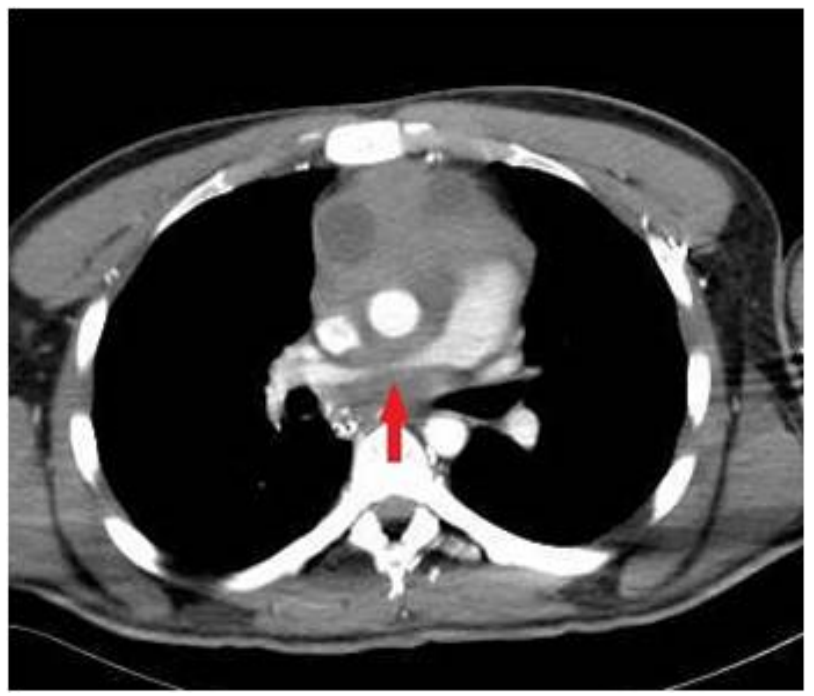

Figure 2. Extrinsic compression of the pulmonary artery by the mediastinal mass.

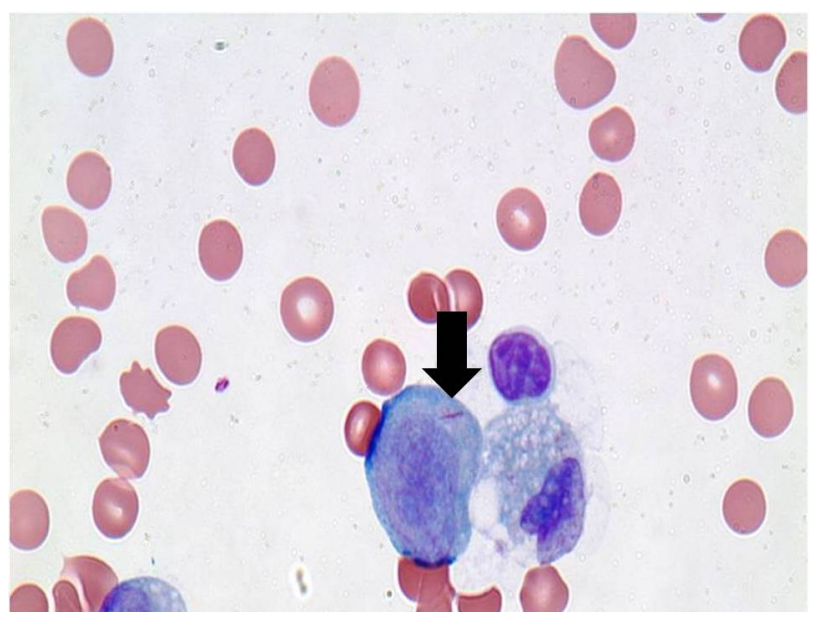

Figure 3. Myeloblast with auer rod.

The patient was started on chemotherapy with a rapid response and with complete resolution of his clinical symptoms. Subsequent echocardiograms showed normalization of his PA pressures and resolution of his right heart dysfunction.

\section{DISCUSSION}

The differential diagnosis of a mediastinal mass is extensive. An unusual cause of a mediastinal mass is myeloid sarcoma, an extramedullary manifestation of acute myeloid leukemia. The diagnosis of acute myeloid leukemia is typically made after a patient presents with constitutional symptoms, i.e. shortness of breath, weight loss, fatigue, and fever.

Further examination reveals an abnormal white blood cell count, either high or low. Evaluation of the white blood cells by peripheral blood smear suggests the diag- nosis, with definitive diagnosis obtained after a bone marrow biopsy. Flow cytometry and special stains are performed to differentiate acute myelogenous leukemia from acute lymphocytic leukemia and to determine leukemia subtype. Rarely, does acute myelogenous leukemia present with soft tissue masses, or granulocytic sarcomas.

It was first described by Burns in 1811. In 1853, King gave the tumor the name, chloroma. Chloroma describes the green color that is characteristic of the tumor, due to myeloperoxidase present within the mass. It was Dock, in 1893, who made the connection between granulocytic sarcomas and acute my eloid leukemia [1]. The chloroma was renamed granulocytic sarcoma by Rappaport, to describe any tumorous mass associated with the granulocytic leukemias [2].

Myeloid sarcomas are present in only $2 \%-8 \%$ of acute myeloid leukemias $[3,4]$. They can be either symptomatic or asymptomatic, depending on the site, and can occur as an isolated finding or in conjunction with acute myeloid leukemia. The usual sites of the occurrence of these sarcomas include skin and lymph nodes, but they have been found to invade bone, epidural structures, soft tissue and GI tract. A recent report of 32 cases and review of the literature showed a poor prognosis in patients with granulocytic sarcoma, either de novo, or when presenting simultaneously with acute myeloid leukemia with a median survival of $9.51+/-19.38$ months [1].

While about a dozen cases have been reported of myeloid sarcoma invading the cardiovascular structures, only one other patient has been recorded to have right heart failure. However, the myeloid sarcoma was diagnosed on post-mortem examination of the patient [5]. To our knowledge, our patient represents the only reported case of myeloid sarcoma causing compressive stenosis of the pulmonary artery with right heart failure with resolution of symptoms after treatment.

\section{REFERENCES}

[1] Paydas, S., Zorludemir, S. and Ergin, M. (2006) Granulocytic sarcoma: 32 cases and review of the literature. Leukemia \& Lymphoma, 47, 2527-2541. doi:10.1080/10428190600967196

[2] Byrd, et al. (1995) Extramedullary myeloid cell tumors in acute nonlymphocytic leukemia: A clinical review. Journal of Clinical Oncology, 13, 1800-1816.

[3] Liu, P.I., Ishimaru, T., McGregor, D.H., et al. (1973) Autopsy study of granulocytic sarcoma (chloroma) in patients with myelongenous leukemia. Hiroshima-Nagasaki 1949-1969. Cancer, 31, 948-955. doi:10.1002/1097-0142(197304)31:4<948::AID-CNCR2 $\underline{820310428>3.0 . C O ; 2-\mathrm{N}}$

[4] Ravandi-Kashani, F., Cortes, J. and Giles, F.J. (2000) Myelodysplasia presenting as granulocytic sarcoma of mediastinum causing superior vena cava syndrome. Leu- 
kemia \& Lymphoma, 36, 631-637.

doi:10.3109/10428190009148412

[5] Tillawi, I.S. and Variakojis, D. (1990) Refractory right ventricular failure due to granulocytic sarcoma. Archives of Pathology \& Laboratory Medicine, 114, 983-985. 